\title{
Slot Architecture for Separating Satellites in Sun-Synchronous Orbits
}

\author{
Karl D. Bilimoria* \\ NASA Ames Research Center, Moffett Field, California 94035 \\ and \\ Rogier A. Krieger ${ }^{\dagger}$ \\ Delft University of Technology, 2629 HS Delft, The Netherlands
}

\begin{abstract}
A slot architecture is developed for separating satellites in congested Sun-synchronous orbits that are a limited natural resource in low Earth orbit. A key feature of the slot architecture is a phasing rule that provides sufficient separation between slots in the vicinity of their orbital crossing points. Other features involve the discretization of orbital parameters such as semi-major axis and right ascension of the ascending node. The design parameters of the slot architecture were selected with detailed consideration of various operational aspects and orbital perturbations. A preliminary analysis indicates that slotmaintenance requirements are automatically satisfied by maneuvers that are routinely performed by current high-value Earth observation satellites to satisfy their existing mission objectives. A simulation analysis of satellites currently in Sun-synchronous orbit confirms that implementation of the proposed slot architecture would greatly reduce the frequency of close approaches between these satellites.
\end{abstract}

\section{Introduction}

$\mathrm{I}_{\mathrm{c}}^{\mathrm{n}}$ n Earth orbit there are currently about a thousand active satellites, plus over 20,000 debris objects with characteristic dimension over $10 \mathrm{~cm}$, hundreds of thousands of debris objects with characteristic dimension over $1 \mathrm{~cm}$, and millions of tiny pieces of debris. ${ }^{1,2}$ The majority of space objects are in low Earth orbit (LEO), i.e., below $\sim 2,000 \mathrm{~km}$ altitude. This frequently results in close approaches or conjunctions which sometimes result in actual collisions between LEO space objects, e.g., the Iridium-Cosmos collision in February 2009. Many Earth observation satellites operate in a congested region of LEO to utilize the desirable illumination properties of Sunsynchronous orbits (SSO) ${ }^{3}$ and there is a risk of collisions among these satellites. For example, a close approach of about $100 \mathrm{~m}$ was predicted between NASA's Cloudsat satellite and the Iranian satellite Sinah-1 in July 2007, causing Cloudsat to perform a collision avoidance maneuver. ${ }^{4}$

Current rules of space operations permit an operator to deploy satellites into any non-geostationary orbit without requiring consideration of other satellites operating in the vicinity. This paper develops a slot architecture designed to maintain separation between SSO satellites, building on a concept proposed in Ref. 5 and explored in Ref. 6. Satellite constellations designed for other purposes have been reported in the literature; for example, the Walker constellation $^{7}$ was designed to provide good coverage of the Earth's surface with a limited number of satellites. The slot architecture developed in this paper is a type of constellation designed with the objective of separating SSO satellites. Its structure is significantly more complex than that of the assigned-longitude slot architecture currently used to separate satellites in geostationary orbit.

Section II below describes some key properties of SSO. An analysis of the current SSO satellite population, including conjunctions (close approaches), is presented in Section III. Details of the slot architecture are presented in Section IV along with a simulation assessment of its performance.

\footnotetext{
${ }^{*}$ Research Scientist, Flight Trajectory Dynamics and Controls Branch, Mail Stop 210-10; Karl.Bilimoria@nasa.gov. Associate Fellow, AIAA.

${ }^{\dagger}$ M.Sc. Student, Faculty of Aerospace Engineering, Kluyverweg 1; r.a.krieger@student.tudelft.nl.
}

1

American Institute of Aeronautics and Astronautics 


\section{Sun-Synchronous Orbits}

Earth observation satellites often use Sun-synchronous orbits because they provide uniform Earth illumination conditions by maintaining a near-constant angle between their orbital plane and the Sun-Earth line; see Fig. 1. The key parameter of a SSO - its constant Sun angle - is typically expressed by a mean local time (MLT) at the orbit's ascending node. MLT is closely related to the angle between the orbit's line of nodes and the Earth-Sun line; this angle remains constant for a SSO. With Sun angles in Fig. 1 measured clockwise relative to the Sun-Earth line, zero angle corresponds to noon, $90 \mathrm{deg}$ to $6 \mathrm{am}, 180 \mathrm{deg}$ to midnight, and $270 \mathrm{deg}$ to $6 \mathrm{pm}$. In order to provide a constant Sun angle, the satellite's orbital plane (or equivalently the line of nodes) must precess at a rate of one revolution per solar year, i.e., $(360 \mathrm{deg} / 365.24$ days $)=0.9856 \mathrm{deg} / \mathrm{day}$.

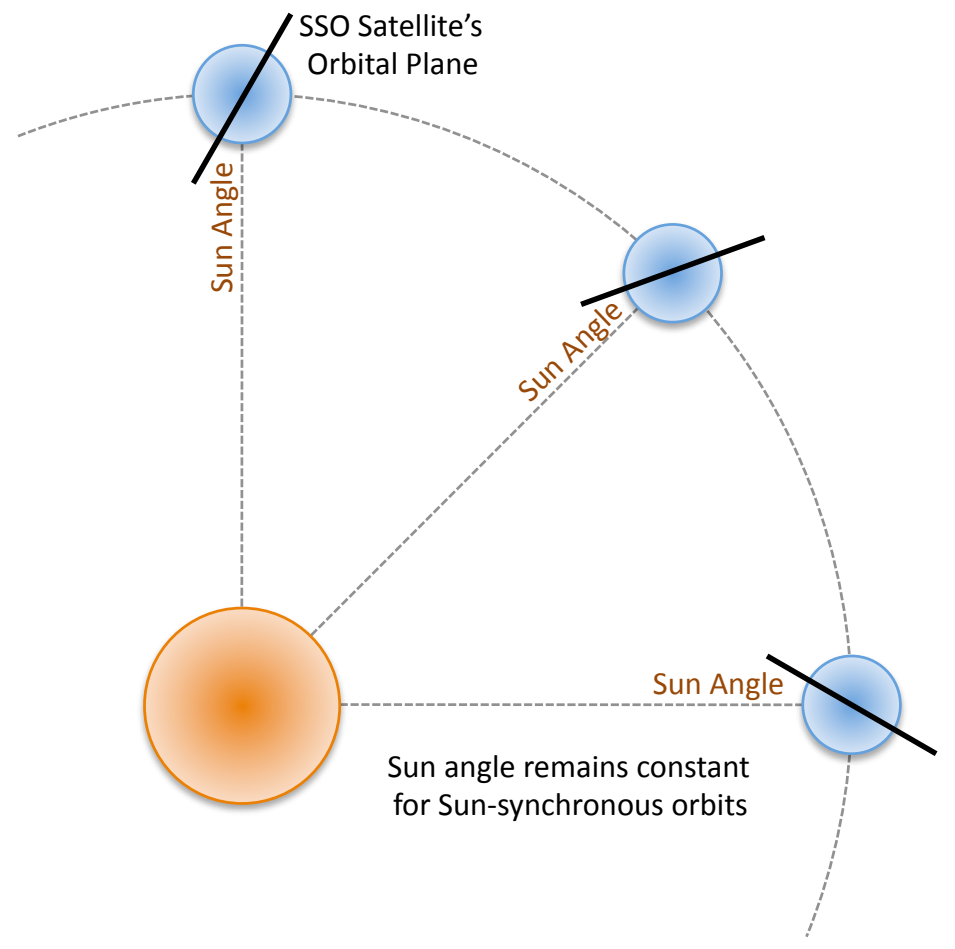

Figure 1. Sun-synchronous orbital plane precession

The Earth's non-uniform gravity field naturally creates an orbital-plane precession rate that depends on the orbital inclination (i), semi-major axis $(a)$, and eccentricity $(e)$. The primary contribution to this motion can be described by an equation ${ }^{8}$ that approximates the rate of change of $\Omega$, right ascension of the ascending node (RAAN). Note that $\Omega$ is also known as longitude of ascending node, and it is closely related to the Sun angle.

$$
\dot{\Omega}=\frac{-3 J_{2}}{2}\left(\frac{R_{\oplus}}{a\left(1-e^{2}\right)}\right)^{2} \sqrt{\frac{\mu}{a^{3}}} \cos (i)
$$

where $\mu=398,600.4418 \mathrm{~km}^{3} / \mathrm{sec}^{2}$ is the Earth's gravitational parameter, $J_{2}=0.0010826267$ represents the principal zonal harmonic due to Earth oblateness, and $R_{\oplus}=6378.137 \mathrm{~km}$ is the Earth's equatorial radius. The orbital elements are illustrated in Fig. 2. Rearranging Eq. (1) above yields:

$$
\cos (i)=\left(\frac{-2 \dot{\Omega}}{3 J_{2} \sqrt{\mu}}\right)\left(\frac{1-e^{2}}{R_{\oplus}}\right)^{2} a^{3.5}
$$


For a SSO, we have $\dot{\Omega}_{S S O}=0.9856 \mathrm{deg} / \mathrm{day}=1.991 \times 10^{-7} \mathrm{rad} / \mathrm{sec}$. With this value, Eq. (2) is known as the SSO condition. Sun-synchronous orbits typically have near-zero eccentricity to satisfy the mission requirements of Earth observation satellites. Hence the SSO condition is primarily a relationship between the satellite's inclination and mean orbital altitude $h=a-R_{\oplus}$.

Mean local time is an important characteristic of a SSO satellite; it is closely related to the Sun angle illustrated in Fig. 1. The MLT (in units of hours) at the ascending node is given by:

$$
M L T=\bmod _{24}\left(24 \frac{(\Omega+\delta)}{360}\right) \text { where } \delta=180-360\left(\frac{\text { Days since Vernal Equinox }}{365.242199}\right)
$$

where vernal equinox occurs on March 20 at various times, e.g., at 17:32 UTC in 2010 . In Eq. (3) above, $(\Omega+\delta)$ is the angle (measured from the ascending node) between the line of nodes and the Sun-Earth line. The time since vernal equinox appears in Eq. (3) to account for the precession of the orbital plane because $\Omega$ is defined relative to the vernal equinox direction (see Fig. 2). The mission objectives of a SSO satellite establish its MLT, which then determines the RAAN at the time when the satellite is placed into its orbit.



Figure 2. Illustration of orbital elements (adapted from Wikimedia Commons)

A repeating ground track (RGT) is a common feature of SSO satellites because it is useful for many Earth observation missions. The characteristic RGT property is that the ground track pattern repeats after an integer number of revolutions $(R)$ around the Earth has been completed in an integer number of days $(D)$. For example, Envisat has RGT parameters of 35D501R meaning that its ground track repeats every 35 days and during that time it makes 501 revolutions around the Earth. From Kepler's Third Law, the orbital period $(P)$ is given by

$$
P=\frac{2 \pi}{\sqrt{\mu}} a^{3 / 2}
$$

where $\mu=398,600.4418 \mathrm{~km}^{3} / \mathrm{sec}^{2}, a$ is in units of $\mathrm{km}$, and $P$ is in units of seconds. Noting that one solar day is $86,400 \mathrm{sec}$, the number of revolutions in one day (also known as mean motion) is: $n=(R / D)=(86,400 / P)$. Substituting Eq. (4) and solving for $a$ results in the RGT relationship: 


$$
a=\mu^{1 / 3}\left(\frac{86,400}{2 \pi(R / D)}\right)^{2 / 3}
$$

Over a typical range of LEO altitudes, the mean motion $(R / D)$ lies between $16 \mathrm{rev} /$ day (at $200 \mathrm{~km}$ altitude) and $12 \mathrm{rev} /$ day (at 1,500 km altitude). For example, Envisat has a mean motion of $14.3143 \mathrm{rev} /$ day, which corresponds to a mean orbital altitude of $800 \mathrm{~km}$ (recall that mean orbital altitude is defined by: $h=a-R_{\oplus}$ ). Due to the RGT requirement that both $D$ and $R$ be integers, the solutions to Eq. (5) are discrete rather than continuous. There are many non-unique solutions that need to be removed, e.g., 4D62R and 6D93R are simply multiples of the fundamental solution 2D31R.



Figure 3. Mean altitude ( $h$ ) vs. RGT days $(D)$ for repeating ground track solutions

Figure 3 illustrates unique solutions of Eq. (5) for a band of LEO altitudes. There is a very similar lobe-like solution structure at altitudes above $900 \mathrm{~km}$; at altitudes below $270 \mathrm{~km}$ there is a partial lobe. For any integer number of days $(D)$ there are multiple RGT solutions at various altitudes, with the number of revolutions $(R)$ being smaller for solutions at higher altitudes. If a 1-day RGT is desired, then there are only two solutions available in the altitude band of Fig. 3: at approximately $270 \mathrm{~km}$ (16 rev/day) and approximately $570 \mathrm{~km}$ (15 rev/day). On the other hand, the number of available solutions for a 59-day RGT is very large, which is characteristic of high-primenumber values of $D$. The spacecraft's mission objectives generally determine the values of $D$ and $R$, and the RGT relationship then determines the design altitude of the orbit.

\section{Analysis of Current SSO Satellite Population}

The analysis presented here is based on the Two Line Element (TLE) data set published by Space Track ${ }^{9}$ on 2 September 2010. This TLE set contained records for a total of 14,652 tracked space objects with characteristic dimension greater than $\sim 10 \mathrm{~cm}$. These space objects include active satellites, defunct satellites, spent rocket bodies, explosion fragments, and other types of space debris. From this set we extracted TLEs that were available for 910 of the 1,010 active satellites compiled from the Union of Concerned Scientists (UCS) database ${ }^{10}$ and the CelesTrak satellite catalog. ${ }^{11}$ Of these 910 satellites, there were 462 satellites in low Earth orbit. Of these 462 LEO satellites, 
201 were SSO satellites which in this work are defined as those with an altitude-inclination combination providing a value of $\dot{\Omega}$ within $\pm 5 \%$ of the SSO precession rate $(0.9856 \mathrm{deg} / \mathrm{day})$ plus a few others that are known to be SSO satellites. Based on the above analysis, it is estimated that $44 \%$ of active satellites in low Earth orbit are in SSO.

Figure 4 shows the LEO satellite population with non-SSO satellites in blue and SSO satellites in red; the SSO condition (for zero eccentricity) is indicated by a dashed black curve. It can be seen that SSO inclinations typically lie between 95 and $100 \mathrm{deg}$, corresponding to retrograde orbits. Due to their near-polar inclinations, SSO satellites can provide coverage over nearly all latitudes.



Figure 4. Mean altitude vs. inclination for low Earth orbit satellites, with SSO highlighted

Figure 5 shows the distribution of ascending node MLTs for the 201 SSO satellites analyzed in this work. MLTs at the descending node differ by 12 hours (i.e., am and pm are switched). It can be seen that some MLTs are more in demand than others.

When two space objects move into close proximity, they are said to be in conjunction. Current operational practice for LEO robotic satellites is to routinely conduct a conjunction analysis using a $(25 \times 25 \times 2) \mathrm{km}$ ellipsoidal threat volume which corresponds to $\pm 25 \mathrm{~km}$ in the along-track (forward/back) and cross-track (left/right) directions and $\pm 2 \mathrm{~km}$ in the radial (up/down) direction. ${ }^{12.13}$ This analysis utilizes a basic trajectory propagator such as SGP-4, described in Refs. 14 and 15. If another space object is predicted to be within this ellipsoid at any time over a lookahead period of several $(\sim 5)$ days, a high-precision trajectory propagator (and possibly additional/enhanced surveillance data) is used to check for conjunctions in a much smaller threat volume (a box of $5 \times 5 \times 0.5 \mathrm{~km}$ ). If a conjunction is still predicted, then a covariance analysis is conducted to determine the probability of collision. If this probability is above a threshold (order of 1 in 1,000) then the satellite is commanded to conduct a collision avoidance maneuver if it has on-orbit propulsive capability. The current operational process effectively assumes that if no conjunctions are predicted in a $(25 \times 25 \times 2) \mathrm{km}$ threat volume then the collision probability is negligible for practical purposes. Hence in this work, a LEO robotic satellite is considered to be safely separated whenever its $(25 \times 25 \times 2) \mathrm{km}$ threat volume is clear of other space objects. 


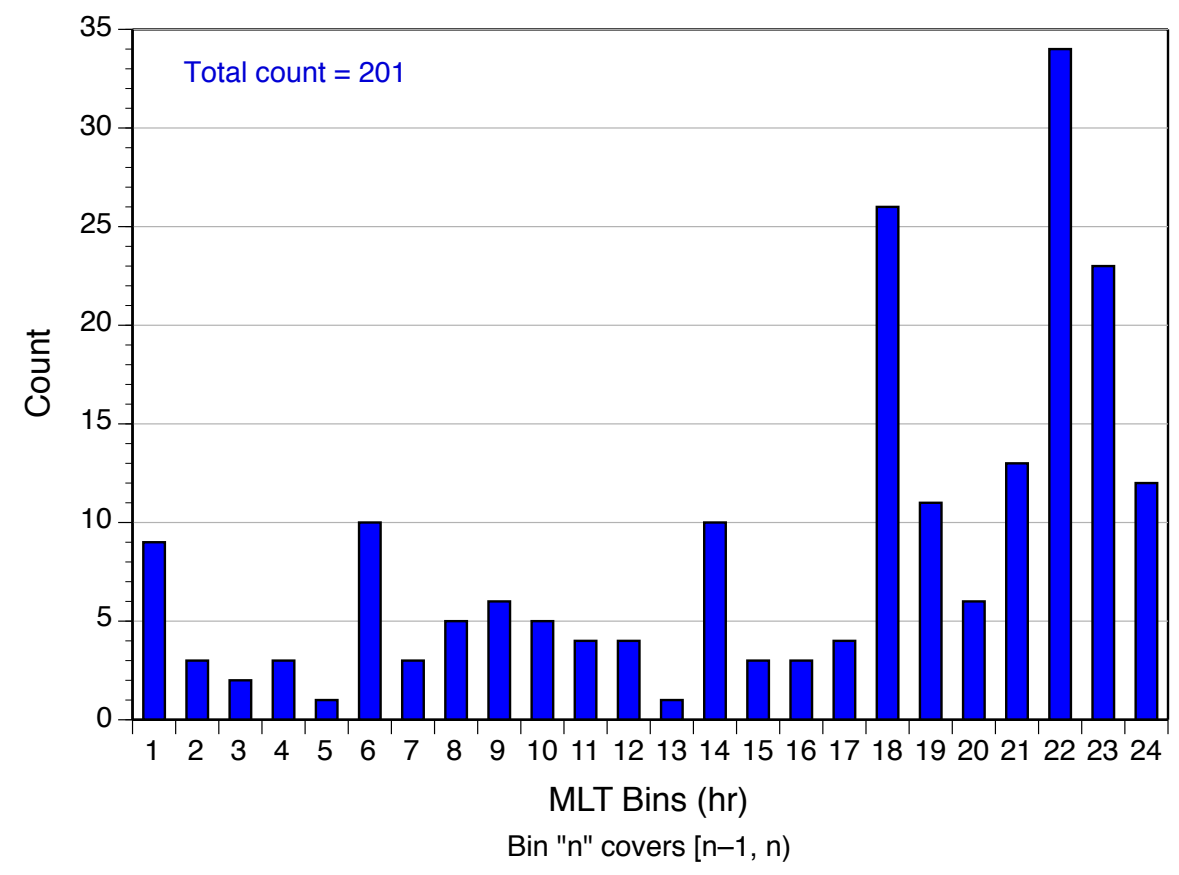

Figure 5. Distribution of mean local times at ascending node for SSO satellites



Figure 6. Approach angle vs. relative speed for SSO satellites with conjunctions in a $(25 \times 25 \times 2) \mathrm{km}$ zone 
The set of 201 SSO satellites were processed for conjunctions among themselves, using the "AdvCAT" conjunction analysis tool in the Satellite Toolkit $(\mathrm{STK})^{16}$ with a $(25 \times 25 \times 2) \mathrm{km}$ threat volume. Over a look-ahead period of 5 days, the conjunction analysis predicted a total of 103 unique conjunctions involving 61 unique satellites corresponding to $30 \%$ of the SSO population. Figure 6 shows the approach angle and relative speed for these conjunctions. Analysis indicates that most of the conjunctions occurred with either high approach angle and high relative speed $(\sim 45 \%)$ or low approach angle and low relative speed $(\sim 35 \%)$. Many of the 103 conjunctions involved a few pairs of satellites going in and out of shallow-angle conjunctions over several successive orbits.

\section{Slot Architecture}

The objective of this work is to develop a slot architecture for separating SSO satellites to eliminate the conjunctions reported in Section III above. The goal is ensure that all SSO satellites are always separated from each other by at least a $(25 \times 25 \times 2) \mathrm{km}$ ellipsoidal volume. The technical approach is to first design a constellation of slots with simplified orbital dynamics such that the slots are guaranteed to be well separated: at least $200 \mathrm{~km}$ in the along-track and cross-track directions, and $30 \mathrm{~km}$ in the radial direction. The rationale for these slot separation distances will be presented later in this section. Each SSO satellite is assigned a slot that satisfies its mission requirements, but after being launched into this slot it will generally drift relative to the slot in accordance with the orbital perturbation dynamics actually experienced by the satellite. When the satellite drift exceeds a pre-defined threshold, it performs a slot-maintenance maneuver to reverse its prior motion relative to the slot. Details are provided below.

\section{Vertical Separation}

To ensure adequate vertical (radial) separation, a series of altitudes (called flight levels) was constructed by adding buffers to the $2 \mathrm{~km}$ radial separation standard to account for various orbital dynamics effects. In this work, the instantaneous orbital altitude is defined as the distance above an Earth-centered sphere with radius equal to $R_{\oplus}$.

The Earth's oblateness results in a non-uniform gravity field that creates (among other effects) a perturbation in the orbital semi-major axis (a) which affects instantaneous orbital altitude. The primary component of this perturbation arises from a short-periodic motion due to the $J_{2}$ term, which creates an oscillation in the value of $a$ with period equal to one-half of the orbital period $(P)$ where $P$ is on the order of 100 minutes. For small orbital eccentricity, we have ${ }^{8}$

$$
\Delta a_{S P} \approx\left(\frac{3 J_{2} R_{\oplus}^{2} \sin ^{2} i}{2 a}\right) \cos 2(\omega+v)
$$

where $v$ is the true anomaly and $\omega$ is the argument of perigee. The amplitude of this short-period perturbation in semi-major axis is on the order of $12 \mathrm{~km}$ for typical SSO parameters.

Another cause of periodic variations in instantaneous orbital altitude, even for a fixed value of $a$, is non-zero orbital eccentricity. Earth observation satellites generally need to maintain a constant orbital altitude, but the $J_{2}$ term results in a rotation of the line of apsides which causes variations in the argument of perigee resulting in altitude perturbations. Hence these satellites often use "frozen orbits" that are characterized by small-amplitude coupled oscillations in eccentricity and argument of perigee. The primary dynamics of this coupled motion can be represented by the $J_{2}$ and $J_{3}$ zonal harmonic terms. The frozen-orbit oscillations in $(e, \omega)$ space occur around the point ( $e \approx 0.001, \omega=90 \mathrm{deg}$ ), and SSO satellites generally target these orbital parameters. ${ }^{8}$ Even a very small eccentricity of 0.001 results in a difference of about $15 \mathrm{~km}$ between apogee and perigee for an orbit with a mean altitude of $1,000 \mathrm{~km}$.

Hence in order to assure a minimum vertical separation of $2 \mathrm{~km}$ between satellites, we need a buffer of $12 \mathrm{~km}$ to compensate for perturbations in semi-major axis plus another buffer of $15 \mathrm{~km}$ to compensate for the small but nonzero eccentricity of a frozen orbit. Adding and rounding up, the radial separation between flight levels is set at 30 $\mathrm{km}$. In this work, the SSO slot-controlled region spans the altitude band from 270 to $900 \mathrm{~km}$, corresponding to the RGT lobes in Fig. 3; this is also the altitude band that contains over $96 \%$ of the current SSO satellite population. Hence there are 22 flight levels spaced $30 \mathrm{~km}$ apart, covering an altitude band of $630 \mathrm{~km}$. The flight-level structure effectively discretizes the semi-major axis orbital parameter $(a)$. 


\section{Horizontal Separation}

To ensure adequate horizontal separation at a given flight level, the slot architecture discretizes two additional orbital parameters. One of these parameters is right ascension of the ascending node $(\Omega)$. As explained in Section II, this orbital parameter is closely related to MLT at the ascending node; see Eq. (3). MLT maintenance requirements for actively controlled current SSO satellites are on the order of 5 minutes. In this work the MLT space is discretized into a grid of 15 minutes which provides, at each flight level, 96 orbital planes that are uniformly spaced by $(360 / 96)=3.75$ deg in RAAN. This family of orbits has numerous intersections in the vicinity of the North and South poles. For example, if the orbital inclination is $97 \mathrm{deg}$, then the orbital paths will intersect at various points along the circles of latitude at $83 \mathrm{deg}$ North and South.

In order to assure adequate separation between slots in neighboring orbital planes, the slots need to be properly phased so that they arrive at their polar-region crossing point at slightly different times. This phasing corresponds to a discretization of the true anomaly $(v)$ at some reference time (the 2010 vernal equinox, in this work). Based on analysis and testing, the following phasing rule was selected to define a set of primary slots:

$$
v=2 \Omega
$$

This phasing rule is illustrated in Fig. 7 where an orange dot shows the primary slot in orbital planes (grey curves) that are spaced 15 minutes apart in MLT (3.75 deg RAAN spacing). Two secondary slots are defined in each orbital plane, placed at $+2.5 \mathrm{deg}$ and $+5 \mathrm{deg}$ true anomaly relative to each primary slot defined by Eq. (6). Consider an orbital plane with $\Omega=30 \mathrm{deg}$. At the reference time, the primary slot in this orbital plane is at $v=60 \mathrm{deg}$ and the two secondary slots are at $v=62.5 \mathrm{deg}$ and $65 \mathrm{deg}$. The neighboring orbital plane has $\Omega=33.75 \mathrm{deg}$ and the primary slot in that plane is at $v=67.5 \mathrm{deg}$ which corresponds to a $2.5 \mathrm{deg}$ true anomaly spacing relative to the last slot $(v=65 \mathrm{deg})$ in the previous $(\Omega=30 \mathrm{deg})$ orbital plane. Therefore at a given flight level there are $(96 \times 3)=288$ slots which are uniformly spaced by $2.5 \mathrm{deg}$ in true anomaly. By design, the motion of these slots is governed by simplified orbital dynamics. STK-based analysis using the point-mass two-body orbital dynamics model shows that the minimum horizontal separation among this constellation of 288 slots is about $240 \mathrm{~km}$. Further analysis indicates that this $240 \mathrm{~km}$ separation would be preserved if the 288 slots were distributed over 48 orbital planes (30 min MLT grid) with six (primary plus five secondary) slots in each plane; i.e., there is a design trade-off between MLT grid size and the number of slots at each MLT. In this work, we use a MLT grid of 15 min with three slots in each orbital plane. Satellites are expected to maintain their MLT within \pm 5 min.

Figure 8 shows a global perspective of the full (three slots per orbital plane) constellation at a single flight level. Although the visual pattern of instantaneous slot positions resembles the seam on a baseball, the motion of the slots is along the orbital paths indicated by grey curves. Figure 9 shows a similar perspective of primary slots colorcoded at three different flight levels. Because the orbital velocity of slots varies with altitude, the baseball-seam patterns at the various flight levels appear to slowly rotate relative to each other over time.

\section{Satellite Motion Relative to Slot}

A slot is an imaginary point whose motion is governed by simplified orbital dynamics: two-body motion plus orbital plane precession due to $J_{2}$. Each slot has constant altitude (semi-major axis) corresponding to one of the flight levels defined earlier and constant inclination whose value is related to the flight level by the SSO condition of Eq. (2). Each slot is associated with a fixed MLT, hence its orbital RAAN varies linearly with time at the SSO rate of $0.9856 \mathrm{deg} / \mathrm{day}$.

Although a satellite is assigned to a slot, its actual motion is affected by all experienced orbital dynamic effects including atmospheric drag, solar radiation pressure, non-uniform Earth gravity field, and lunar-solar perturbations. Hence there will be some relative motion between the satellite and its assigned slot. Since SSO satellites operate in LEO, atmospheric drag effects are a key factor governing satellite motion relative to its slot. The drag causes a decay in orbital altitude, and the rate of decay increases as altitude decreases. For example, at an altitude of $600 \mathrm{~km}$ (middle of the flight level band) the altitude decay rate is on the order of $1.5 \mathrm{~km} / \mathrm{year} .{ }^{17}$ As the satellite's altitude decreases, its velocity increases according to orbital dynamics. Since the slot by definition remains at its original assigned flight level, the satellite gradually moves ahead of its slot as its altitude decays. 


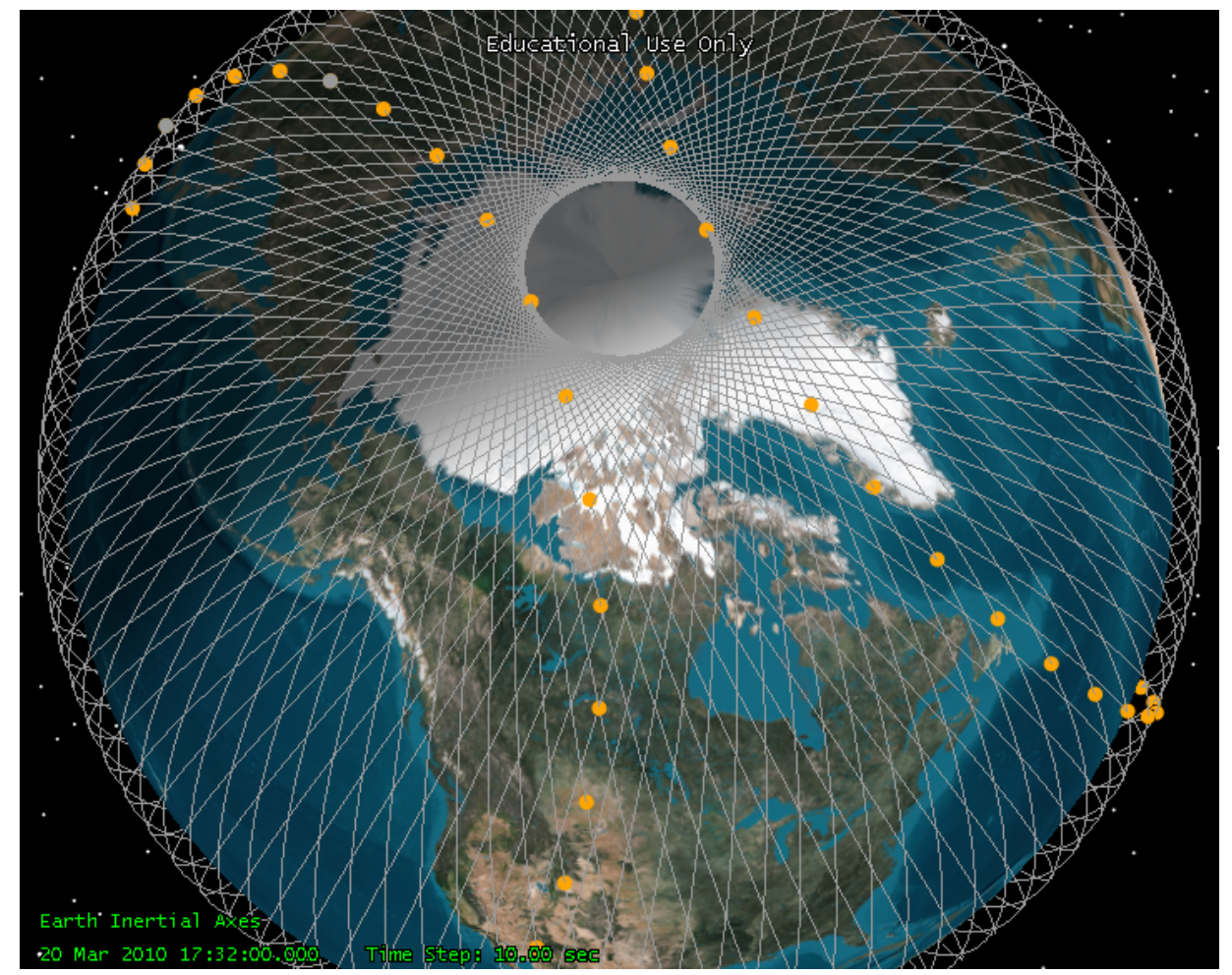

Figure 7. Polar-region perspective of primary slots in their orbital planes at a single flight level

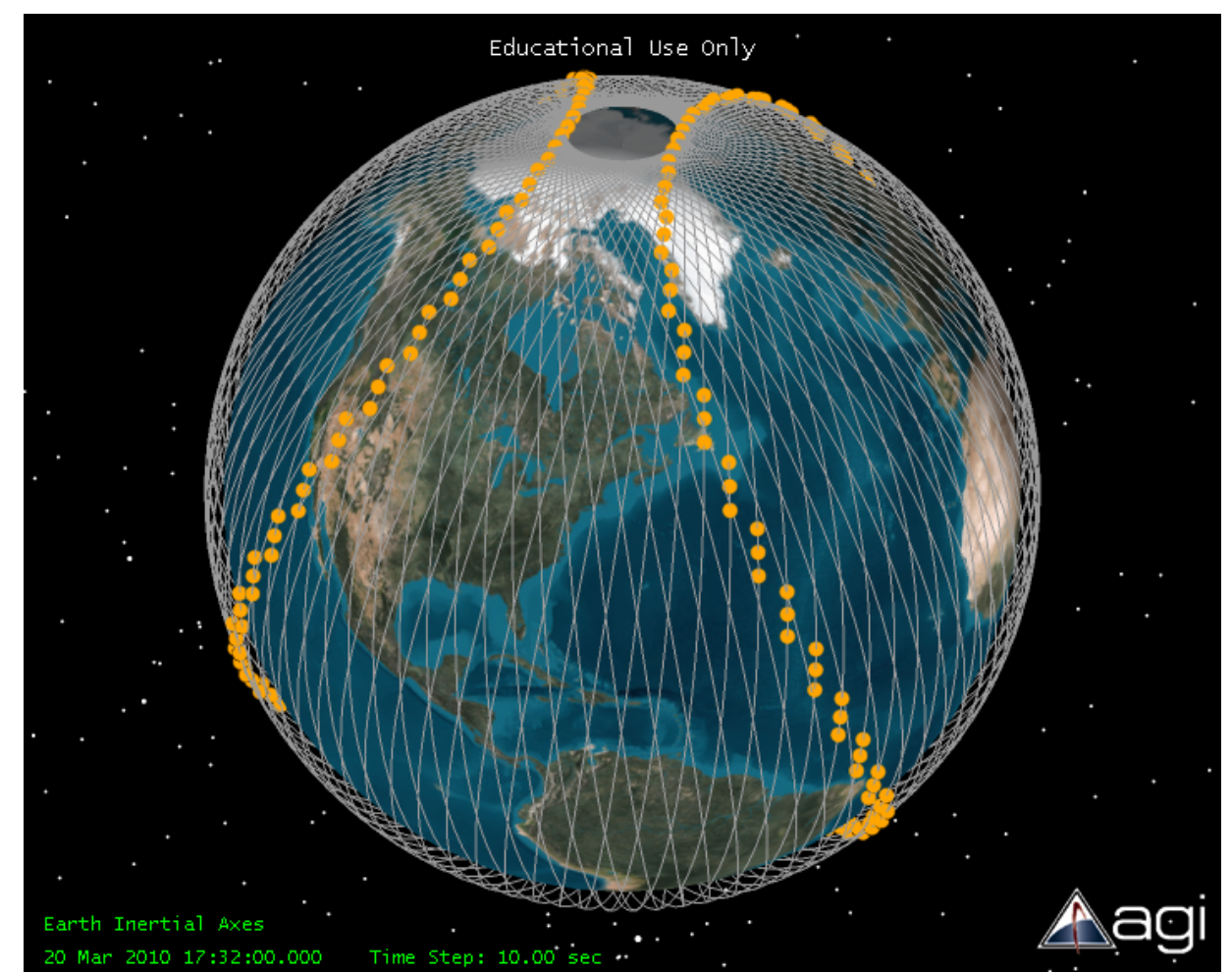

Figure 8. Global perspective of full complement of three slots per orbital plane at a single flight level 


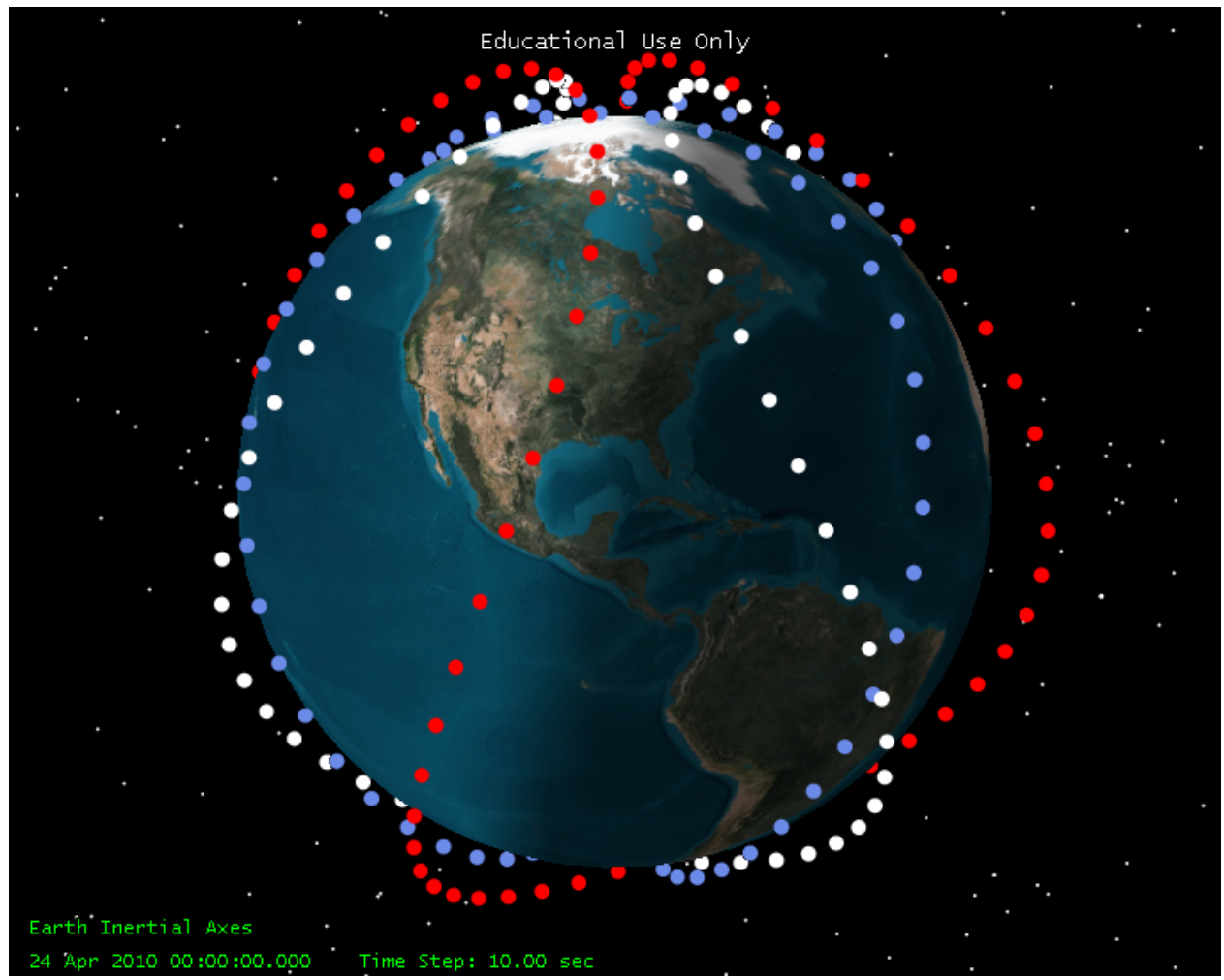

Figure 9. Primary slots at three different (color-coded) flight levels

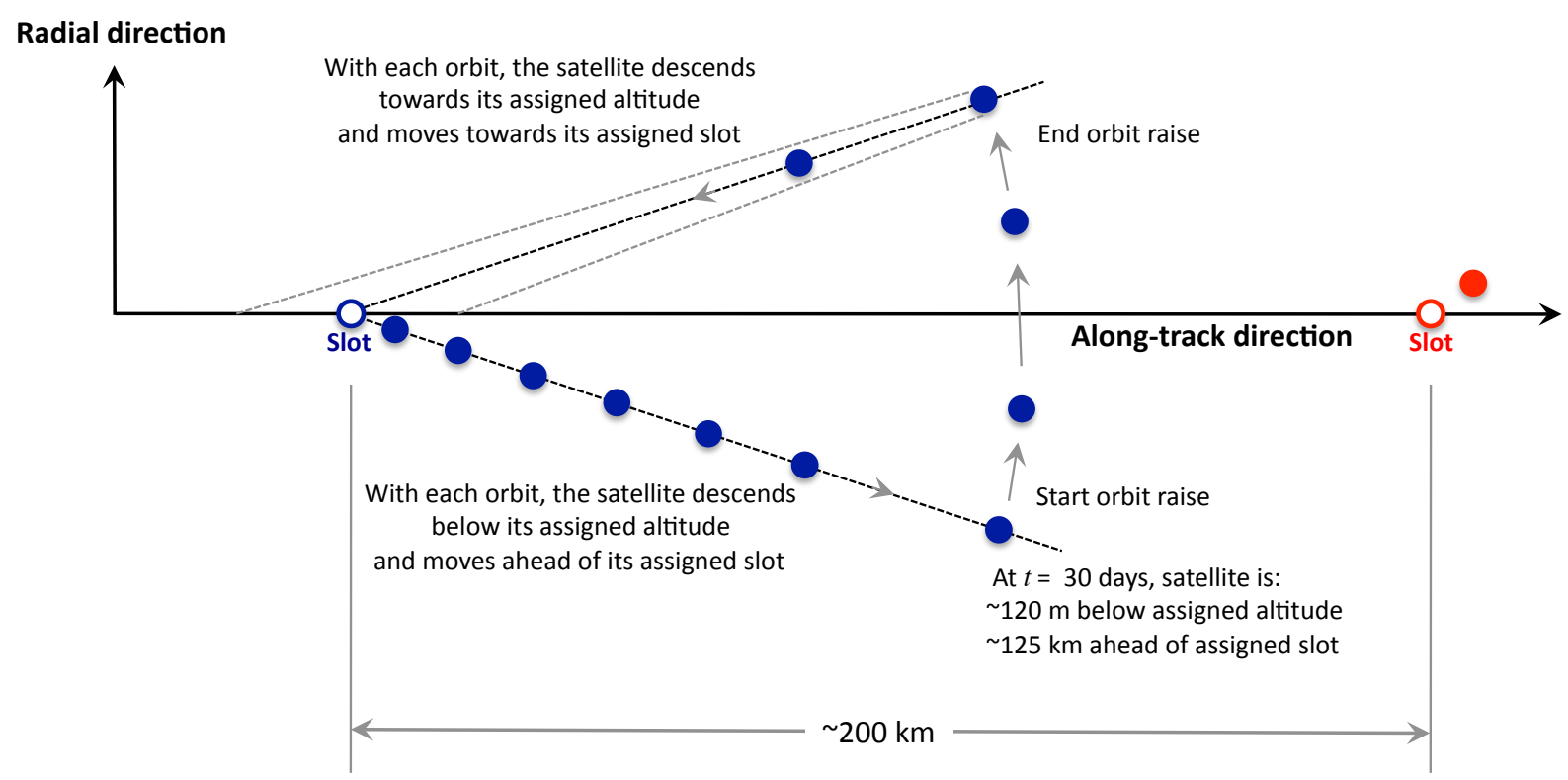

Figure 10. Example of drag-induced motion of a satellite relative to its slot 
A rough-order-of-magnitude analysis indicates that over a period of 30 days, a satellite at $600 \mathrm{~km}$ altitude will descend by about $120 \mathrm{~m}$ due to atmospheric drag effects and will therefore move about $125 \mathrm{~km}$ ahead of its slot. Recall that the slots are horizontally separated by at least $240 \mathrm{~km}$ in the vicinity of their polar-region crossing points. STK-based analysis showed that some of this separation buffer may be used up by an allowed \pm 5 min MLT drift (caused by RAAN drift relative to SSO rate) of the satellite relative to its slot. In a worst-case MLT-drift scenario, the $240 \mathrm{~km}$ separation is reduced to $200 \mathrm{~km}$ (for a satellite at $600 \mathrm{~km}$ altitude). In an even more challenging scenario, a satellite that has moved $125 \mathrm{~km}$ ahead of its slot may find itself $(200-125)=75 \mathrm{~km}$ behind a satellite assigned to a slot in a neighboring orbital plane (equivalently, a neighboring MLT). This $75 \mathrm{~km}$ distance is still 50 $\mathrm{km}$ more than the $25 \mathrm{~km}$ along-track dimension of the threat volume typically used to protect robotic LEO satellites. Hence even for a very challenging (and rare) scenario, there will be adequate horizontal separation after a 30-day along-track drift of a satellite relative to its assigned slot. This scenario is depicted in Fig. 10. To compensate for the drift, the satellite must periodically perform a slot maintenance maneuver. In the above example scenario, the satellite performs an altitude raise maneuver after it moves about $125 \mathrm{~km}$ ahead of its slot; during this maneuver it moves from $\sim 120 \mathrm{~m}$ below its assigned slot to $\sim 120 \mathrm{~m}$ above that altitude. When the satellite is at a higher altitude than its slot, it moves slower than the slot and hence the along-track relative motion brings it back towards the slot. When the satellite descends to its assigned altitude it will be close to its slot's along-track position; when it descends below the assigned altitude it will begin moving forward relative to its slot. Since the forward and backward relative motions both occur over a roughly 30-day interval, the altitude raise maneuver needs to be performed once every 60 days. Note that if the neighboring slot (hollow red circle in Fig. 10) were unoccupied, we could allow a larger along-track drift and the altitude-raise maneuver could be performed less frequently than 60 days.

To gain some perspective about slot maintenance maneuvers, we studied the orbit maintenance maneuvers routinely performed by some currently operational satellites. Recall that in the above example scenario, the satellite raises its altitude after 60 days when its altitude drops about $120 \mathrm{~m}$ below its assigned slot altitude. As examples of current SSO satellite operations, here are some observations derived from historical TLE data covering the one-year period Aug 2009 - 2010: Envisat (nominal altitude $800 \mathrm{~km}$ ) maintained altitude within $\pm 15 \mathrm{~m}$ by performing an orbital maneuver on a $\sim 30$-day cycle; Cloudsat (nominal altitude $700 \mathrm{~km}$ ) maintained altitude within $\pm 75 \mathrm{~m}$ by performing a orbital maneuver on a $\sim 60$-day cycle; and, RapidEye-1 (nominal altitude $625 \mathrm{~km}$ ) maintained altitude within $\pm 75 \mathrm{~m}$ by performing an orbital maneuver on a $\sim 90$-day cycle. Note that these orbit maintenance maneuvers are performed to satisfy basic requirements for the satellites' Earth observation mission, and that these requirements are more stringent than the slot-maintenance maneuvers described above. Hence in many cases the slot maintenance requirements are automatically satisfied by maneuvers that are already performed routinely to satisfy mission objectives. Note that many current SSO satellites (e.g., nanosatellites) do not have the capability to perform orbital maneuvers; however this capability would be a requirement for satellites operating in the proposed slot-controlled SSO zone in the altitude band of $270-900 \mathrm{~km}$.

\section{Simulation of Slot Architecture}

An STK-based simulation was run to test the separation performance of the proposed slot architecture. The active SSO satellite population found in the 2 Sep 2010 TLE set was mapped to a slot architecture with the discretization parameters described above. On the available grid of altitude and MLT, the satellites were assigned to the grid point closest to their altitude and MLT values as found in the TLE data. Only satellites with TLE-derived altitudes between 270 and $900 \mathrm{~km}$ were assigned to slots; consequently eight of the $201 \mathrm{SSO}$ satellites were excluded from this simulation. At each $(h, M L T)$ grid point, there were three slots available at different values of true anomaly. Each of the 22 flight levels had a total of 288 slots, for a grand total of 6,336 available slots. Despite the large number of available slots, a few high-demand $(h, M L T)$ combinations had more requests than the available three slots. In such cases, priority was given to satellites that had orbital maneuvering capability. Consequently 12 nanosatellites, which had no maneuvering capability, could not be accommodated in their desired $(h, M L T)$ grid point. Each of these nanosatellites was assigned to a slot that provided their desired flight level at a neighboring MLT. Constellations of SSO satellites, such as the NASA A-Train and RapidEye, are intended to fly in close proximity by station-keeping among themselves; hence each constellation of satellites was assigned to a single slot. From the original total of 201 SSO satellites, the simulation included 171 satellites: eight satellites were excluded because their altitude was outside the slot-controlled SSO zone and 34 satellites were consolidated within 12 constellation slots. The simulation was initialized with the orbital parameters $(a, i, e, \omega, \Omega, v)$ of these 171 satellites adjusted (relative to their values in the TLE data) to the corresponding values of their assigned slots. 


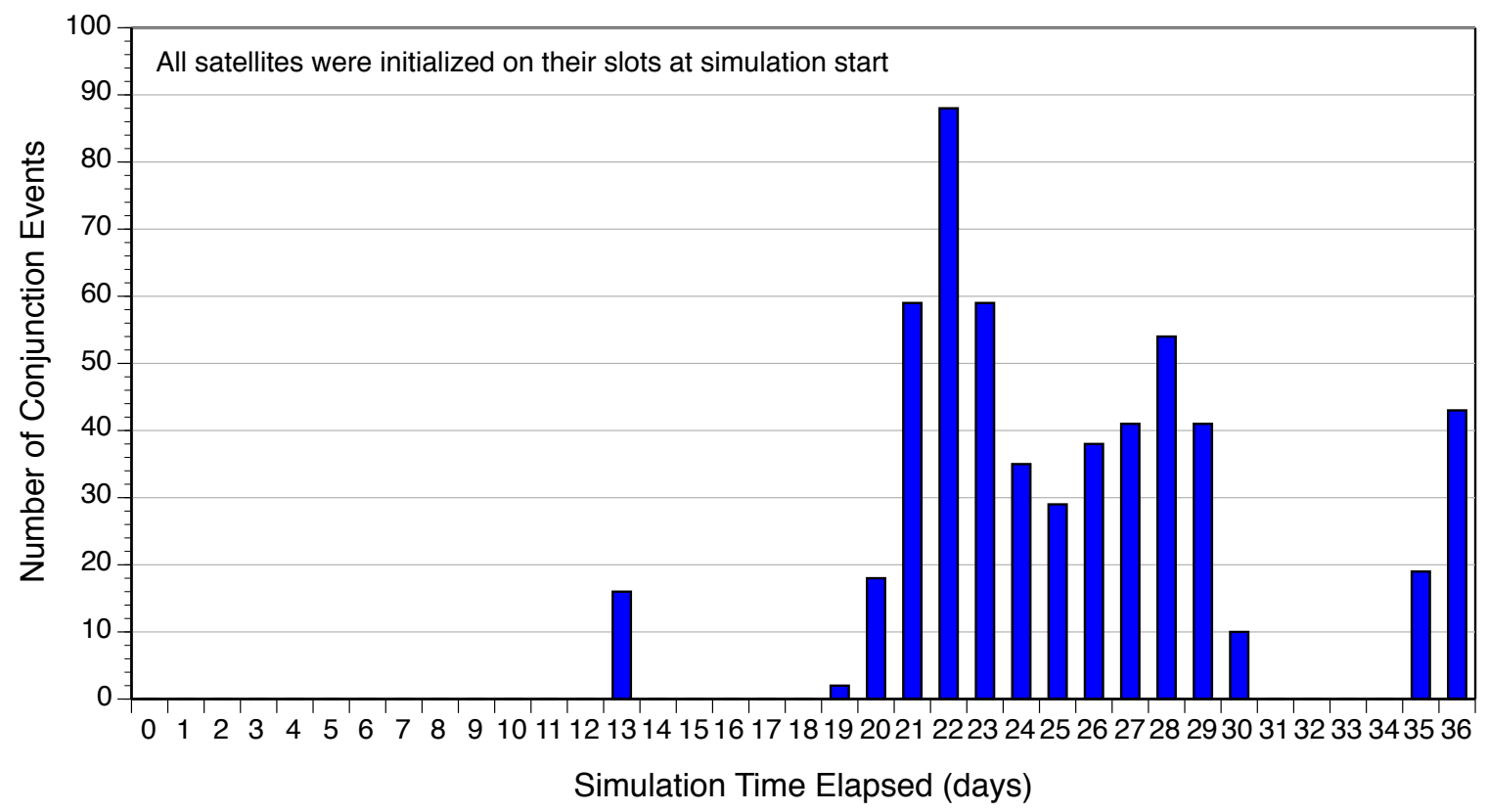

Figure 11. Temporal distribution of conjunction events

These 171 SSO satellites were analyzed for conjunctions among themselves, using the STK "AdvCAT" conjunction analysis tool with a $(25 \times 25 \times 2) \mathrm{km}$ threat volume. Recall that a previous conjunction analysis (see Section III) of 201 SSO satellites without slot allocation found a total of 103 unique conjunctions, involving 61 unique satellites, over a look-ahead period of 5 days; the earliest conjunction was predicted to occur during the first day. With the slot allocation scheme implemented as described above, no conjunctions were found over a 5-day look-ahead period.

Although the accuracy of orbital trajectory predictions using SGP-4 starts to decrease beyond a 7-day look-ahead period, the conjunction analysis was performed again with a look-ahead period of 36 days. Over this time period of 36 days, only 16 of the 171 simulated satellites were found to be in conjunction with each other due to the cumulative effects of orbital perturbations that caused them to drift relative to their assigned slots. The total number of conjunction events (including many that involved a few pairs of satellites going in and out of conjunction over several successive orbits) over the 36-day simulation period was 552. A temporal distribution of these conjunction events is shown in Fig. 11. It can be seen that conjunctions began appearing regularly 19 days into the simulation; prior to that there was only conjunction, on Day 12, involving a satellite with unusually high drag properties. Noting that the simulation began with the satellites located directly in their assigned slots, the 19-day period is in reasonable agreement with the 30-day slot-conformance period estimated in the rough-order-of-magnitude analysis presented earlier (see Fig. 10).

This initial simulation exercise did not have the capability to model slot maintenance maneuvers. Future work will expand the fidelity and capability of the simulation to verify that there will be no conjunctions if the satellites perform these maneuvers when necessary.

\section{Concluding Remarks}

A slot architecture has been developed for separating satellites in Sun-synchronous orbits. The approach taken is to first design a constellation of slots with simplified orbital dynamics such that the slots are guaranteed to be well separated. A key feature of the slot architecture is a phasing rule that provides sufficient separation of slots at their near-polar crossing points. Each SSO satellite is assigned a slot that satisfies its mission requirements, and will generally drift relative to its slot in accordance with the orbital dynamics experienced by the satellite. When the satellite drift exceeds a pre-defined threshold, it performs a slot-maintenance maneuver to reverse its prior motion relative to the slot. The design parameters for the slot architecture and maintenance maneuvers were developed with careful consideration of operational aspects typical of SSO satellites. A preliminary analysis indicates that slotmaintenance requirements, estimated to be on a cycle of $\sim 60$ days, are automatically satisfied by maneuvers that are 
routinely performed by current high-value Earth observation satellites to satisfy their existing mission objectives. A simulation analysis of satellites currently in Sun-synchronous orbit confirms that implementing the proposed slot architecture would greatly reduce the frequency of close approaches between these satellites.

\section{Acknowledgments}

The authors are grateful to Will Marshall, Creon Levit, and James Mason at NASA Ames and Michael Singer at University of California - Santa Cruz for their valuable comments and suggestions during the course of this work. Rogier Krieger would like to thank STC and STIEP for their financial support of this research work performed at NASA Ames Research Center.

\section{References}

${ }^{1}$ Limiting Future Collision Risk to Spacecraft: An Assessment of NASA's Meteoroid and Orbital Debris Programs, National Research Council, The National Academies Press, Washington, D.C., 2011.

${ }^{2}$ Klinkrad, H., Space Debris: Models and Risk Analysis, Springer-Verlag, New York, 2006.

${ }^{3}$ Boain, R.J., "The A-B-Cs of Sun-Synchronous Orbit Mission Design," Paper No. 04-108, 14 ${ }^{\text {th }}$ AAS/AIAA Space Flight Mechanics Conference, February 2004.

${ }^{4}$ Orbital Debris Quarterly News, NASA Orbital Debris Program Office, Vol. 11, Issue 4, October 2007.

${ }^{5}$ Anilkumar, A.K., et al, "Space Traffic Management," International Space University, Space Studies Program, Beijing, China, 2007. URL: http://www.isunet.edu/index.php?option=com_docman\&task=doc_download\&gid=371 [cited 19 Jan 2011 ].

${ }^{6}$ Weeden, B. and Shortt, K., "Development of an Architecture of Sun-Synchronous Orbital Slots to Minimize Conjunctions," Paper No. 2008-3547, AIAA SpaceOps 2008 Conference, May 2008.

${ }^{7}$ Walker, J.G., "Satellite Constellations," Journal of the British Interplanetary Society, Vol. 37, 1984, pp. 559-571.

${ }^{8}$ Vallado, D.A., Fundamentals of Astrodynamics and Applications, 3rd ed., Springer-Microcosm Press, New York, 2007.

${ }^{9}$ United States Strategic Command, Space Track: The Source for Space Surveillance Data. URL: http://www.space- track.org [cited 19 Jan 2011]

${ }^{10}$ Union of Concerned Scientists, UCS Satellite Database. URL: http://www.ucsusa.org [accessed 13 August 2010].

${ }^{11}$ Center for Space Standards and Innovation, CelesTrak. URL: http://celestrak.com [accessed August 2011].

${ }^{12}$ Newman, L.K. and Frigm, R.C., "NASA CARA Process, System, and Experiences Overview," Conjunction Summary Message (CSM) Users Conference, Fairfax, VA, July 2010. URL: http://www.space-track.org/csm_workshop.html [cited 25 Jan 2011]

${ }^{13}$ Klinkrad H., Alarcon, J.R., and Sanchez, N., "Collision Avoidance for Operational ESA Satellites," Proceedings of the Fourth European Conference on Space Debris (ESA SP-587), Darmstadt, Germany, 2005.

${ }^{14}$ Hoots, F.R. and Roehrich, R.L., "Spacetrack Report No. 3: Models for Propagation of NORAD Element Sets," U.S. Air Force Aerospace Defense Command, Colorado Springs, Colorado, 1980.

${ }^{15}$ Vallado, D.A., Crawford, P., Hujsak, R., and Kelso, T.S., "Revisiting Spacetrack Report \#3," Paper No. 2006-6753, AIAA/AAS Astrodynamics Specialist Conference, 2006.

${ }^{16}$ Satellite Toolkit Software Package, version 9.2.0, Analytical Graphics Incorporated, Exton, PA, 2009.

${ }^{17}$ Wertz, J. and Larson, W., Space Mission Analysis and Design, $3^{\text {rd }}$ ed., Microcosm Press, Hawthorne, CA and Springer, New York, 1999. 